\title{
LA FORMACIÓN DEL PROFESORADO Y ALUMNADO UNIVERSITARIO EN EL USO DE TIC Y MOODLE
}

\author{
Marisol Rodríguez Correa \\ Universidad Internacional de La Rioja \\ Juan Carlos Rivadulla López \\ Universidade da Coruña
}

\begin{abstract}
RESUMEN: Las TIC desempeñan un fuerte papel motor en todos los sectores de nuestras sociedades, por lo que nuestras Universidades deberán adaptarse a las posibilidades y potencialidades que ofrecen estas herramientas. El objetivo principal de este estudio es conocer la formación en TIC y Moodle de los principales agentes protagonistas del sistema educativo universitario y sus necesidades formativas. Para ello se llevó a cabo una investigación con una metodología cuantitativa de carácter no experimental de tipo encuesta en la que participaron 190 alumnos y 46 profesores de la Facultad de Ciencias de la Educación de la Universidade da Coruña. Los resultados de este estudio evidencian que aunque la mayoría del profesorado y alumnado consultado poseen conocimientos y competencias digitales sobre los recursos tecnológicos e informáticos, presentan algunas carencias, existiendo la necesidad de formación para el desarrollo de ciertas competencias como la "búsqueda de información digital" y "el manejo del ordenador". Además, en cuanto a la valoración que realizan acerca de la formación recibida para el uso de las TIC y del Moodle, ambos colectivos consultados consideran de la misma forma, que no poseen los conocimientos necesarios para el uso adecuado de estas herramientas.
\end{abstract}

PALABRAS CLAVE: Formación, entorno virtual, universidad, e-learning, teleformación, TIC.

\section{TRAINING OF UNIVERSITY TEACHERS AND STUDENTS IN THE USE OF ICT AND MOODLE}

ABSTRACT: ICT plays a strong leading role in all sectors of our societies, so that our universities must be adapted to the possibilities and potential offered by these tools. The main objective of this study is to incorporate ICT training and Moodle as the main protagonists of the university's education 
system agents and their training needs. It was investigated with a quantitative nonexperimental methodology type of survey in which 190 students and 46 teachers of the Faculty of Education at the University of Corunna participated. The results of this study showed that although the majority of teachers and students consulted possess knowledge and expertise on digital technology and information resources, some gaps are present. Training develop certain skills in the "pursuit of digital information" and "computer management". Furthermore, with regard to the assessment made about such training for the use of ICT and Moodle, both groups surveyed -considered in the same manner that-, they do not possess the knowledge required for the proper use of these tools.

KEYWORDS: Training, virtual environment, university, e-learning, virtual learning, ICT.

Recibido: 23/02/2016

Aceptado: 17/05/2016

Correspondencia: Marisol Rodríguez Correa, Universidad Internacional de La Rioja, Facultad de Educación. Calle Islas Cíes, 3,2A, La Coruña.15190. Email: mrodriguez@udc.es.

\section{INTRODUCCIÓN}

La incorporación de las TIC en todos los niveles del sistema educativo ha conformado una nueva cultura tecnológica que ha generado importantes esfuerzos en cuanto a adaptación a estos nuevos medios (Rodríguez-Correa y González-Sanmamed, 2013). De esta forma, se ha creado una conciencia generalizada acerca de la necesidad de formación para el uso de estas herramientas tanto por parte del profesorado como del alumnado, para lograr integrarlas de manera significativa a los procesos de enseñanza aprendizaje (Cabero y Marín, 2014).

La principal función de este tipo de formación sería desarrollar en el docente y el alumno/a un sentido crítico en el uso y aprendizaje de los medios tecnológicos. Por tanto, existe la necesidad de vincular la teoría con la práctica, el feedback continuo y el perfeccionamiento día a día de las actividades que se desarrollarán en este tipo de propuestas formativas.

El Moodle es un sistema de gestión de cursos (Course Management SystemCMS-) que facilita a los docentes crear comunidades de aprendizaje en línea, basándose en una pedagogía constructivista, la cual afirma que el conocimiento se construye en la mente del estudiante en base a sus habilidades y conocimientos propios, en vez de simplemente publicar y transmitir la información que se considera que el mismo debe conocer.

Con la incorporación de las TIC y de las plataformas de teleformación como el Moodle en las Instituciones de Educación Superior, nos encontramos ante la necesidad de nuevos modelos que ayuden a los profesores a afrontar la docencia explotando las posibilidades de estas tecnologías (utilización de lenguajes más icónicos, 
requerimientos distintos de presentación de la información, cambios en las funciones de los docentes, cambios en los modelos comunicativos, etc.) (Salinas, 2002; Rodríguez-Correa, Iseni y Varela, 2013; Gómez Galán, 2014). Por ello, la Universidad española está poniendo en práctica innumerables acciones de innovación educativa, con el fin de superar los esquemas didácticos tradicionales y lograr una enseñanza de calidad, objetivo fundamental del proceso de convergencia europea que se está viviendo en la actualidad (Álvarez, Cuéllar, López, Adrada, Anguiano, Bueno, Comas, y Gómez, 2011).

Frecuentemente nos encontramos con algunos profesores que muestran resistencia al cambio e incluso a la idea de incorporar algunas TIC y plataformas de teleformación a sus prácticas. Según Selwyn, Marriot y Marriot (2002) algunas de estas resistencias tendrán que ver en algún grado con las carencias de culturas institucionales en cuanto a la utilización de las TIC. Sin embargo otros estudios señalan por el contrario, que los profesores universitarios tienen actitudes positivas para la utilización e incorporación de las TIC en los procesos de formación (Jimoyiannis y Komis, 2007; Banas, 2010; Alvarez y otros, 2011; Guzmán, García y Chaparro, 2011; Sang, Valcke, van Braak, Tondeur, y Zhu, 2011; Gutiérrez, 2014).

Para disminuir estas resistencias, el docente deberá recibir una capacitación conceptual respecto a cómo las TIC y las plataformas de teleformación se comportan en los ambientes educativos, la cual ayudará a transformar las creencias que tengan respecto a estos medios, y le permitirá tomar decisiones para adaptar y buscar nuevas formas de utilización, y no ser meramente un reproductor de prácticas de enseñanzas elaboradas por otros (Cabero, 2014). Esta formación del profesorado deberá contemplar por lo tanto, un enfoque más global e integrador en el que se tenga en cuenta las dimensiones instrumental, pragmática, didáctica, metodológica, psicológica, actitudinal, investigadora y curricular (Del Moral y Villalustre, 2010); de esta forma, "se garantizará no solo la adquisición de competencias relacionadas con el uso y el manejo de herramientas tecnológicas (destrezas, habilidades, dominio de softwares, etc.), sino competencias personales vinculadas al proceso de aprendizaje del alumno y de enseñanza del profesorado" (Muñoz-Repiso, Gómez-Pablos, González, Martín, Rodero, Martín y Marcos, 2015, p. 86).

En los últimos años, numerosas investigaciones han arrojado en sus resultados que la formación del profesorado en TIC no ha sido la más adecuada (Balanskat, Blamire y Kefala, 2006; Becta, 2004; Cabero, 2004; Condie y Munro, 2007; Galanouli, Murphy y Gardner, 2004; Llorente, 2008; Waite, 2004; Almerich, Suarez, Belloch y Bo, 2011; Diez, 2012), ya que por lo general se ha focalizado más en el dominio de los recursos tecnológicos, siendo olvidada la formación del componente pedagógico.

Con respecto a los alumnos/as la visión que se les suele ofrecer a los mismos/ as en su formación inicial en TIC, tanto en la Universidad como durante su período de prácticas, se acerca demasiado al enfoque instrumental, el cual reduce estos medios a artefactos concretos y condiciona la construcción del pensamiento de este colectivo acerca de las TIC (Noelle y Granados, 2015). Por otra parte, la formación que reciben para el uso de plataformas de teleformación es escasa, el alumnado no suele tener competencias en Moodle e incluso algunos no saben lo que es (Henríquez y Ugel, 2012). 
Gutiérrez, Palacios y Borrego (2010) afirman en base a los resultados obtenidos en su estudio sobre la formación de los futuros maestros y la integración de las TIC en educación, que "el carácter innovador de las nuevas herramientas, nuevos modos y nuevos lenguajes de comunicación en manos del alumnado; la posibilidad de considerar las TIC como medios de expresión creativa, de participación democrática, quedan muy lejos de las percepciones y expectativas de los actuales estudiantes..." (291).

Nos encontramos igualmente ante una necesaria formación del alumno/a para el uso las TIC y de las plataformas de teleformación, la cual promoverá en el mismo/a una actitud crítica del uso de estos medios, logrando de esta forma no solo su manejo, sino también una calidad de la interacción tanto con los contenidos como con las otras personas que las utilizan en el transcurso de las prácticas educativas en las que se implica (Barberá y Badia, 2008).

Los resultados de algunos estudios han confirmado, como las actitudes ante las TIC por parte de los profesores que reciben formación mejoran significativamente respecto a los que no la reciben (Gilmore, 1998). De esta forma, los profesores que promueven más el uso de materiales electrónicos en la enseñanza universitaria, suelen ser aquellos con mayores niveles de formación, con mayores destrezas tecnológicas y quienes se perciben más comprometidos con la enseñanza (MacDowell, 2002).

Consideramos tal y como lo señalan Olcott y Wright (1995), que existe una necesidad urgente de alfabetización tecnológica, que implicará una formación basada en metodologías educativas aplicadas a la tecnología y nuevos modelos de evaluación, los cuales deberían ser requisitos indispensables para todos los docentes.

Indudablemente esta formación continua para el uso de TIC y de plataformas de teleformación del profesorado universitario y del alumnado, conllevaría a generar de la misma forma, cambios en la forma de pensar, juzgar y sobre todo de actuar de ambos. Es importante destacar que la formación de los docentes y alumnos/as ante el uso de estas herramientas, es uno de los principales factores que podría incidir en el éxito o fracaso de los proyectos educativos.

Según Bates (2005) los profesores sólo cambiarán si pueden ver claramente los beneficios del cambio y las desventajas de no cambiar. Para ello, el docente necesitará desarrollar nuevas habilidades de tipo técnico, es decir, una capacitación o actualización en el manejo de ciertas TIC, como ordenadores e internet y otras habilidades basadas en el diseño didáctico haciendo parte a la tecnología de su práctica docente. El reto estaría en que las Universidades innoven además del área tecnológica sus concepciones y prácticas pedagógicas y de formación, modificando por lo tanto el modelo de enseñanza universitario.

Abordar este proceso significaría reformular el papel y práctica pedagógica tanto del docente como del alumno/a, "planificar y desarrollar modelos de aprendizaje del alumnado radicalmente distintos a los tradicionales, cambiar las formas organizativas del tiempo y el espacio de las clases, cambiar las modalidades y estrategias de tutorización... (Area, 2005, p. 3), y ofrecer una formación sólida para el uso de las TIC y de las plataformas de teleformación para ambos protagonistas de la acción educativa. 


\section{Método}

\section{Objetivos}

En este estudio se recogen las valoraciones de un grupo de estudiantes y docentes universitarios acerca de la formación en TIC y más concretamente de la plataforma Moodle. Así, se han planteado los siguientes objetivos tanto para el profesorado como para el alumnado:

- Determinar los conocimientos y competencias digitales que poseen.

- Identificar la formación que tienen en TIC y en Moodle, así como sus necesidades formativas.

- Analizar las valoraciones del alumnado o profesorado sobre la formación que necesita el profesorado o alumnado sobre las TIC y Moodle.

\section{Población y muestra}

El estudio se realizó con alumnado y profesorado de la Facultad de Ciencias de la Educación de la Universidade da Coruña (UDC). Las condiciones para colaborar fueron que los participantes usaran la plataforma Moodle en la Universidad y que hubiesen tenido más de cuatro asignaturas con apoyo en la plataforma estudiada. Por estos dos motivos, en cuanto al alumnado se escogió como población objeto de estudio a los 240 estudiantes matriculados en segundo y tercer curso del grado de Educación Primaria, aunque la muestra final fue de 190 participantes (79.2\%), y en cuanto al profesorado se escogió como población objeto de estudio a los 54 profesores que imparten docencia en segundo y tercer curso del grado de Educación Primaria, aunque la muestra final fue de 46 participantes (85.2\%).

Somos conscientes de que cualquier investigación requiere que la muestra represente a un subconjunto de la población objeto de estudio (Buendía, Colás y Hernández, 1997). No obstante, esta afirmación debe ser entendida en el marco de las finalidades y objetivos del estudio. Por ello, podemos establecer que la muestra cumple con los criterios de calidad para obtener respuestas suficientes, válidas y representativas (Scheaffer, Mendenhall y Lyman, 2007). Así, el error muestral que estamos dispuestos a cometer con la muestra tomando como población de referencia al estudiantado y profesorado de la Facultad de Ciencias de la Educación del último curso del que se disponen datos, es de $3.25 \%$ y de $5.61 \%$ respectivamente (tabla 1 ).

Tabla 1. Error muestral

\begin{tabular}{lccc}
\cline { 2 - 4 } & $\begin{array}{c}\text { Población de partida. } \\
\text { Curso 2014-2015 }\end{array}$ & Muestra & $\begin{array}{c}\text { Error } \\
\text { muestral }(*)\end{array}$ \\
\hline $\begin{array}{l}\text { Alumnado matriculado en } 2^{\circ} \text { y } 3^{\circ} \text { de } \\
\text { Educación Primaria }\end{array}$ & 240 & 190 & $3,25 \%$ \\
\hline $\begin{array}{l}\text { Profesorado que imparte docencia en } \\
2^{\circ} \text { y } 3^{\circ} \text { de Educación Primaria }\end{array}$ & 54 & 46 & $5,61 \%$ \\
\hline
\end{tabular}

(*) Valores obtenidos a partir de: http://www.mdk.es/esp/errores.html. 
En cuanto a las características de los participantes, con relación al alumnado la mayoría son mujeres (75.3\%) de entre 20 y 25 años (47.9\%). Además, todos poseen ordenador en propiedad, sobre todo un dispositivo portátil (66.8\%), y la inmensa mayoría (88.4\%) utiliza el sistema operativo Windows (tabla 2). En referencia al profesorado, la mayoría también son mujeres (63\%) de entre 51 y 60 años $(39.1 \%)$. Todos poseen ordenador en propiedad, tanto de sobremesa como portátil $(58.7 \%)$, y el sistema operativo que utilizan es Windows (78.3\%).

Tabla 2. Características de la muestra

\begin{tabular}{|c|c|c|c|c|}
\hline \multicolumn{3}{|c|}{ Variables } & \multirow{2}{*}{$\begin{array}{c}\begin{array}{c}\text { Alumnado } \\
(\mathbf{n}=\mathbf{1 9 0})\end{array} \\
143(75.3 \%)\end{array}$} & \multirow{2}{*}{$\begin{array}{c}\begin{array}{c}\text { Profesorado } \\
(\mathbf{n}=\mathbf{4 6})\end{array} \\
29(63 \%)\end{array}$} \\
\hline \multirow{8}{*}{ 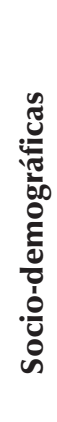 } & \multirow{2}{*}{ Género } & Mujer & & \\
\hline & & Hombre & $47(24.7 \%)$ & $17(37 \%)$ \\
\hline & \multirow{6}{*}{ Edad } & $18-20$ & $87(45.8 \%)$ & -- \\
\hline & & $20-25$ & $91(47.9 \%)$ & -- \\
\hline & & $26-30$ & $12(6.3 \%)$ & -- \\
\hline & & $31-40$ & -- & $8(17.4 \%)$ \\
\hline & & $41-50$ & -- & $16(34.8 \%)$ \\
\hline & & $51-60$ & -- & $18(39.1 \%)$ \\
\hline \multirow{9}{*}{ 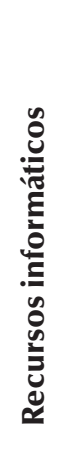 } & & Más de 60 & -- & $4(8.7 \%)$ \\
\hline & \multicolumn{2}{|c|}{ Ordenador en propiedad } & $190(100 \%)$ & $46(100 \%)$ \\
\hline & \multirow{3}{*}{ Tipo de ordenador } & Portátil & $127(66.8 \%)$ & $19(41.3 \%)$ \\
\hline & & Portátil y sobremesa & $59(31.1 \%)$ & $27(58.7 \%)$ \\
\hline & & Otros & $4(2.1 \%)$ & \\
\hline & \multirow{4}{*}{ Sistema operativo } & Windows & $168(88.4 \%)$ & $36(78.3 \%)$ \\
\hline & & Mac Os & $9(4.7 \%)$ & $10(21.7 \%)$ \\
\hline & & Windows + Mac Os & $9(4.7 \%)$ & -- \\
\hline & & Windows + Linux & $4(2.1 \%)$ & -- \\
\hline
\end{tabular}

\section{Instrumento y procedimiento}

Para le recogida de información se ha utilizado un cuestionario tipo Likert con cinco niveles de respuesta (muy en desacuerdo; en desacuerdo; ni de acuerdo, ni en desacuerdo; de acuerdo; muy de acuerdo) (García et al., 2014). Este tipo de escalas es frecuente en educación, pues el constructo percepción de competencias está presente en una parte importante de las investigaciones sobre destrezas y capacidades. Se le suele definir como las creencias individuales sobre las capacidades que se poseen en el desarrollo de una tarea determinada o en el ejercicio de una competencia concreta (Usher y Pajares, 2008). 
El cuestionario, tomando como referencia de la investigación de Sánchez, Sánchez y Ramos (2012), está compuesto por 8 dimensiones, presentando en este artículo los resultados alcanzados en las dimensiones 3, 4, 6 y 8, referidos a los "Conocimientos Digitales" (formado por 10 ítems), a las "Competencias Digitales" (formado por 13 ítems), a la "Formación en TIC" (formado por 10 ítems) y a la "Formación en Moodle" (formado por 10 ítems) (tabla 3). Para dar respuesta a la intencionalidad exploratoria y descriptiva de este estudio (Bisquerra, 2004), se ha utilizado una metodología cuantitativa de carácter no experimental de tipo encuesta (McMillan y Schumacher, 2005). Concretamente se utilizó un muestreo no probabilístico denominado "muestreo por conveniencia", teniendo en cuenta la disponibilidad de los participantes o la facilidad de acceso. Los análisis estadísticos se realizaron con el programa SPSS 23, calculando medidas de tendencia central (media), el valor más repetido (moda) y de dispersión (desviación típica).

Para asegurar las condiciones de validez, la primera versión del cuestionario fue sometida a juicio de expertos y a un estudio piloto, tanto con alumnado como con profesorado. En cuanto a la fiabilidad, se ha procedido al análisis del Alpha de Cronbach para cada uno de las cuatro dimensiones (tabla 3), obteniéndose un $\alpha=$ 0,938 en el alumnado y $\alpha=0.874$ en el del profesorado para la dimensión de "Conocimientos Digitales"; un $\alpha=0,807$ en el alumnado y de $\alpha=0.868$ en el profesorado para la dimensión de "Competencias digitales"; un $\alpha=0,836$ en el alumnado y $\alpha=$ 0.950 en el del profesorado para la dimensión de "Formación en TIC" y un $\alpha=0,871$ en el alumnado y de $\alpha=0.889$ en el del profesorado para la última dimensión sobre la "Formación en Moodle".

Tabla 3. Fiabilidad de las escalas

\begin{tabular}{lccc}
\hline \multirow{2}{*}{ Dimensiones } & \multirow{2}{*}{$\mathbf{N}^{\mathbf{*}}$ de ítems } & \multicolumn{2}{c}{ Alpha de Cronbach } \\
\cline { 3 - 4 } & & Alumnado & Profesorado \\
\hline Conocimientos digitales & 10 & 0,938 & 0,874 \\
Competencias digitales & 13 & 0,807 & 0,868 \\
Formación en TIC & 10 & 0,836 & 0,950 \\
Formación en Moodle & 10 & 0,871 & 0,889 \\
\hline
\end{tabular}

La recogida de datos se desarrolló durante el segundo cuatrimestre del curso académico 2014-2015 y para ello se informa a los participantes de que los cuestionarios tendrán un tratamiento única y exclusivamente para el fin de esta investigación y que serán anónimos, solicitándoles la máxima sinceridad en sus respuestas.

\section{Resultados}

Con respecto a los conocimientos que los participantes indican que tienen sobre los recursos tecnológicos e informáticos (tabla 4), se aprecian valores medios bastante elevados, sobrepasando tanto en el alumnado como en el profesorado el valor de 
3 en todos los ítems. Así pues, el profesorado otorga un alto valor al manejo de una cámara de vídeo básica $(4,07)$ y el uso de distintas redes sociales $(4,28)$, aunque estos a mayores también indican que conocen los reproductores de vídeo y DVD $(4,43)$, siendo el valor más repetido (moda) en los tres ítems el 5. Por su parte en el alumnado, sobresalen con una media de 4,23 y una moda de 5 el conocimiento de recursos y dispositivos de uso habitual en el día a día de los jóvenes, como el uso de distintos medios de mensajería instantánea y chat, el manejo de una cámara de vídeo básica y el uso de distintas redes sociales.

En cuanto a los ítems menos valorados, tanto el profesorado como el alumnado coinciden en señalar el poco conocimiento sobre los distintos componentes de un ordenador (3,7 y 3,78 de media respectivamente y una moda de 4) y los distintos dispositivos móviles (3,61 y 3,78 respectivamente y una moda de 5).

En el caso de la desviación típica, el valor mínimo se encuentra en el conocimiento de distintos procesadores de textos, hojas de cálculo... tanto en el profesorado $($,80097) como en el alumnado (,79066), mientras que el valor máximo del profesorado se encuentra en el ítem sobre el conocimiento de Distintos medios de mensajería instantánea, chat $(1,54545)$ y el del alumnado en el conocimiento de distintos dispositivos móviles (1,3177).

Tabla 4. Conocimientos digitales del profesorado y del alumnado

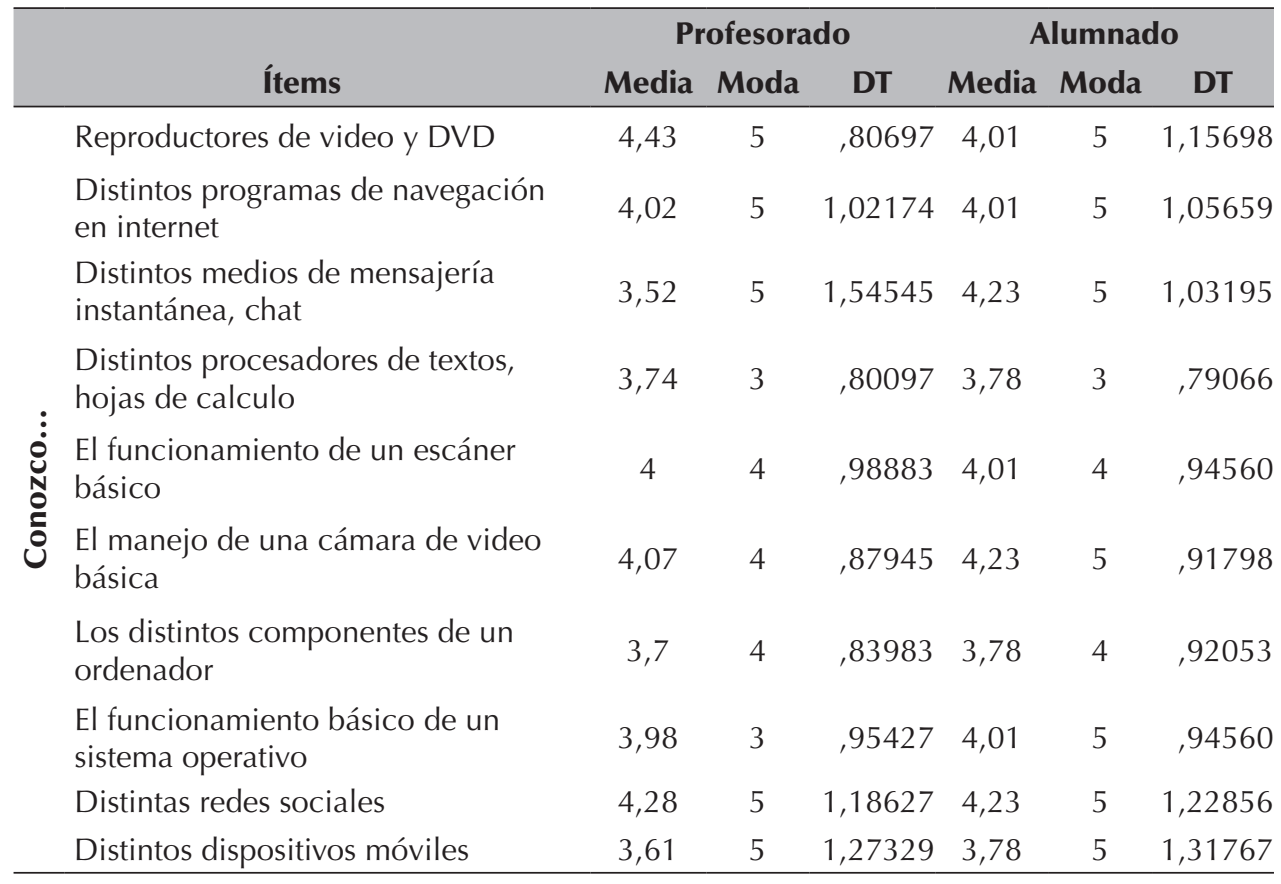

Sobre las competencias digitales (tabla 5), las respuestas promedias son más moderadas; de hecho, tanto en el profesorado como en el alumnado, hay varios ítems cuya media de respuestas se encuentran por debajo del valor 3. Cabe destacar que 
las respuestas de ambos colectivos se encuentran enfrentadas. Así, mientras el profesorado considera que es capaz de utilizar un proyector de presentaciones $(4,93)$, buscar en bases de datos $(4,26)$ y manejar plataformas virtuales de enseñanzaaprendizaje $(4,2)$ (los tres ítems con una moda de 5), el alumnado mayoritariamente se considera capaz de elaborar blogs $(4,18)$, utilizar internet como medio didáctico $(4,07)$ y editar vídeos $(4,07)$ (los dos primeros ítems con una moda de 5 y el tercer con una de 4).

Referente a los ítems menos valorados, el profesorado le otorga poco valor a la capacidad de diseñar páginas web sencillas $(1,83)$, editar vídeos $(2,22)$ y elaborar blogs $(2,59)$ (los tres ítems con una moda de 1), siendo estos dos aspectos últimos los que el alumnado otorgó mucho valor. Por su parte, el alumnado indica el diseño de actividades para plataformas virtuales $(2,75)$ y el diseño de aplicaciones multimedia $(2,97)$, obteniéndose una moda de 1 en ambos casos.

En cuanto a la desviación típica, el valor mínimo en el caso del profesorado se encuentra en la capacidad para utilizar un proyector de presentaciones $(, 24964)$ mientras que en el alumnado en la de elaborar blogs (,85621). El valor máximo en el profesorado es precisamente la elaboración de blogs $(1,75849)$ y en el alumnado el diseño de aplicaciones multimedia $(1,50285)$.

Tabla 5. Competencias digitales del profesorado y del alumnado

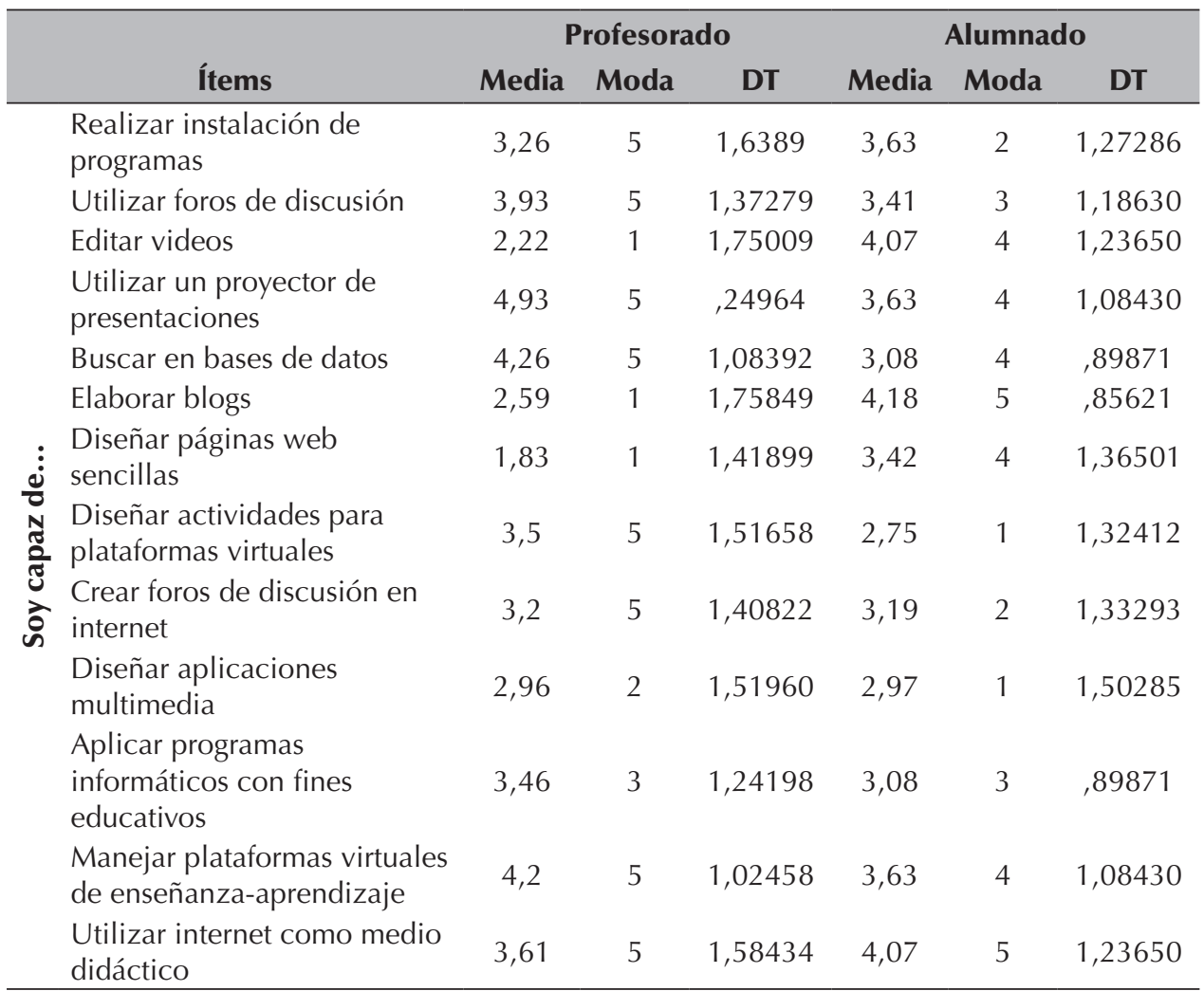


Con relación a la formación en TIC (tabla 6), mientras que el profesorado considera mayoritariamente $(4,52)$ que los conocimientos referidos al uso de las TIC los han adquirido a través de cursos facilitados por la Institución, la media del alumnado que valora este aspecto es muy inferior $(1,92)$. Tanto el profesorado como el alumnado consideran que poseen las habilidades informáticas requeridas para el uso adecuado de instrumentos telemáticos como el correo electrónico, foros y videoconferencias (3,7 y 3,99 respectivamente) y las habilidades didácticas requeridas para el uso adecuado de estos instrumentos (3,87 y 3,48 respectivamente). Además, también consideran que en caso de que en las materias que cursen/impartan sea conveniente usar software y/o hardware específicos, poseen las habilidades requeridas para el uso de los mismos (3,52 y 3,47 respectivamente). Estos hechos contrastan con la valoración que hace un colectivo sobre el contrario, ya que mientras que el profesorado $(4,65)$ considera que el alumnado tiene un nivel de formación adecuado para el uso de las TIC, el alumnado que considera que el profesorado tiene un nivel de formación adecuado es inferior $(3,06)$.

Finalmente, en cuanto a la formación que consideran necesaria recibir sobre $\mathrm{TIC}$, tanto el profesorado como el alumnado indican necesario formarse específicamente en aspectos referidos a la búsqueda de información (revistas, documentos...) (4,13 y 3,58 respectivamente) y sobre el manejo y uso del ordenador $(4,09$ y 3,07 respectivamente). El aspecto que menos valoran es el de recibir formación sobre el correo electrónico (2,74 y 2,75 respectivamente).

El valor que más se repite (moda) en el conjunto de ítems del profesorado es el 5 (en 6 ítems), mientras que en el alumnado es el 4 (5 ítems).

En el caso de la desviación típica, el valor mínimo en el profesorado (,65791) se encuentra en la valoración que hacen sobre si los conocimientos referidos al uso de las TIC han sido adquiridos a través de cursos facilitados por la institución, mientras que el del alumnado (,95789) se encuentra en la valoración de las habilidades didácticas que poseen para el uso adecuado de distintos instrumentos. Mientras, el valor máximo del profesorado $(1,65649)$ se encuentra en el ítem sobre las habilidades requeridas para el uso de software y/o hardware en caso de que sea conveniente en las materias que imparta, y en el caso del alumnado, el valor máximo $(1,49746)$ se encuentra en el requerimiento de formación sobre aplicaciones informáticas básicas (word, excell...).

Con relación a la formación para el uso de la plataforma Moodle, así como la opinión sobre la formación que tiene o debería tener el profesorado o alumnado sobre esta plataforma respectivamente (tabla 7), ambos colectivos consideran mayoritariamente que los conocimientos referidos al uso de Moodle los han adquirido a través de cursos facilitados por la Institución (4,8 y 4,08 respectivamente). Además, tanto el profesorado $(2,78)$ como el alumnado $(2,66)$ consideran que no poseen los conocimientos necesarios para el uso adecuado de Moodle, aspecto que se ve refrendado con la valoración que hace un colectivo sobre el contrario, ya que tanto el profesorado $(2,93)$ como el alumnado $(2,85)$ consideran que el alumnado/profesorado no tiene un nivel de formación adecuado para el uso de Moodle. De hecho, ambos colectivos (4,37 y 4,03 respectivamente) piensan que los otros deberían recibir formación para mejorar sus competencias en Moodle. 
Tabla 6. Formación en TIC

\begin{tabular}{|c|c|c|c|c|c|c|}
\hline \multirow[b]{2}{*}{ Ítems } & \multicolumn{3}{|c|}{ Profesorado } & \multicolumn{3}{|c|}{ Alumnado } \\
\hline & Media & Moda & DT & Media & Moda & DT \\
\hline \multirow{2}{*}{$\begin{array}{l}\text { Los conocimientos referidos al uso } \\
\text { de las TIC han sido adquiridos a } \\
\text { través de cursos facilitados por la } \\
\text { institución. } \\
\text { Poseo las habilidades informáticas } \\
\text { requeridas para el uso adecuado } \\
\text { de instrumentos telemáticos como } \\
\text { el correo electrónico, foros y } \\
\text { videoconferencias, entre otros }\end{array}$} & 4,52 & 5 & 65791 & 3,92 & 4 & ,96678 \\
\hline & 2,7 & 1 & 1,61754 & 2,99 & 3 & 1,15698 \\
\hline \multirow{2}{*}{$\begin{array}{l}\text { En caso de que en las materias que } \\
\text { imparta/curse sea conveniente usar } \\
\text { software y/o hardware específicos, } \\
\text { poseo las habilidades requeridas } \\
\text { para el uso de los mismos } \\
\text { Poseo las habilidades didácticas } \\
\text { requeridas para el uso adecuado de } \\
\text { distintos instrumentos }\end{array}$} & 2,52 & 1 & 1,65649 & 2,47 & 2 & 1,12994 \\
\hline & 2,87 & 1 & 1,52911 & 2,48 & 2 & ,95789 \\
\hline $\begin{array}{l}\text { El manejo y uso del } \\
. \quad \quad \text { ordenador }\end{array}$ & 4,09 & 5 & 1,48812 & 3,07 & 3 & 1,30722 \\
\hline 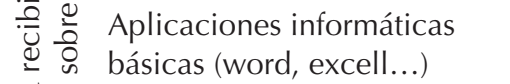 & 4,2 & 5 & 1,54373 & 2,97 & 1 & 1,49746 \\
\hline 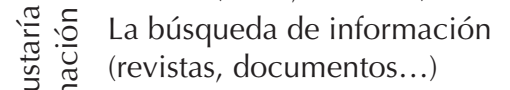 & 4,13 & 5 & 1,54357 & 3,58 & 4 & 1,13191 \\
\hline 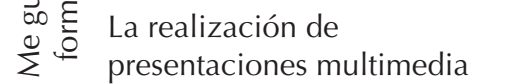 & 4,21 & 5 & ,96409 & 4,02 & 4 & 1,11970 \\
\hline El uso del correo electrónico & 2,74 & 1 & 1,48226 & 2,75 & 4 & 1,16808 \\
\hline $\begin{array}{l}\text { Los docentes/alumnos tienen un } \\
\text { nivel de formación adecuado para el } \\
\text { uso de las TIC }\end{array}$ & 4,26 & 5 & 1,12417 & 2,85 & 4 & 1,08519 \\
\hline
\end{tabular}

Finalmente, en cuanto a las preferencias sobre la formación que les gustaría recibir sobre Moodle, cabe destacar que en términos generales ambos colectivos indican como necesario recibir formación sobre todos los aspectos consultados (subida y bajada de apuntes, envío de documentos, uso de chat/foro, uso de correo electrónico, elaboración de exámenes), ya que la mayoría de los ítems superan la media de 3, excepto uno (el uso del correo electrónico). Concretamente, el profesorado mayoritariamente señala la necesidad de recibir formación sobre cómo realizar los exámenes $(4,22)$ y sobre cómo realizar consultas al alumno $(4,2)$. Mientras que el alumnado indica necesario recibir formación sobre cómo realizar los exámenes $(3,57)$ y sobre el uso de chat/foro $(3,37)$.

El valor que más se repite (moda) en el conjunto de ítems del profesorado es el 5 (en 6 ítems), mientras que en el alumnado es el 3 (7 ítems). 
En cuanto a la desviación típica, el valor mínimo en ambos colectivos se encuentra en la valoración que hacen sobre si los conocimientos referidos al uso de Moodle los han adquirido a través de cursos facilitados por la Institución (,68701 y ,61141 respectivamente). El valor máximo en el profesorado se refiere al requerimiento de formación sobre el envío de documentos $(1,44011)$ y en el alumnado sobre la bajada de apuntes (1,35282).

Tabla 7. Formación en Moodle

\begin{tabular}{|c|c|c|c|c|c|c|}
\hline \multirow[b]{2}{*}{ Ítems } & \multicolumn{3}{|c|}{ Profesorado } & \multicolumn{3}{|c|}{ Alumnado } \\
\hline & Media & Moda & DT & Media & Moda & DT \\
\hline $\begin{array}{l}\text { Los conocimientos referidos al uso } \\
\text { de Moodle han sido adquiridos a } \\
\text { través de cursos facilitados por la } \\
\text { institución. }\end{array}$ & 4,8 & 5 & 68701 & 4,08 & 4 & ,61141 \\
\hline $\begin{array}{l}\text { Poseo las conocimientos necesarios } \\
\text { para el uso adecuado de Moodle }\end{array}$ & 2,78 & 3 & 1,03092 & 2,56 & 3 & 1,10521 \\
\hline \multicolumn{7}{|l|}{$\begin{array}{l}\text { Me gustaría recibir formación } \\
\text { específica de Moodle sobre...: }\end{array}$} \\
\hline La subida/bajada de apuntes & 3,54 & 3 & 1,06888 & 3,11 & 3 & 1,35282 \\
\hline El uso del correo electrónico & 2,46 & 2 & 1,20566 & 2,33 & 3 & 1,17507 \\
\hline El uso de chat/foro & 4,13 & 5 & 1,24023 & 3,37 & 3 & ,76363 \\
\hline El envío de documentos & 3,72 & 5 & 1,44011 & 3,13 & 1 & 1,30142 \\
\hline Cómo realizar los exámenes & 4,22 & 5 & 1,34846 & 3,57 & 3 & 1,0882 \\
\hline $\begin{array}{l}\text { Cómo realizar consultas al docente } \\
\text { / alumno }\end{array}$ & 4,19 & 5 & 1,16656 & 3,03 & 3 & ,92756 \\
\hline \multicolumn{7}{|l|}{ Los docentes/alumnos...: } \\
\hline $\begin{array}{l}\text { Tienen un nivel de formación } \\
\text { adecuado para el uso de Moodle }\end{array}$ & 2,93 & 2 & 1,14314 & 2,85 & 3 & 1,15899 \\
\hline $\begin{array}{l}\text { Deberían recibir formación para } \\
\text { mejorar sus competencias en } \\
\text { Moodle }\end{array}$ & 4,37 & 5 & ,95123 & 4,03 & 5 & 1,04353 \\
\hline
\end{tabular}

\section{Conclusiones y Discusión}

Con nuestro trabajo de investigación hemos pretendido conocer y analizar las valoraciones de un grupo de alumnos/as y profesores de la Facultad de Ciencias de la Educación de la Universidade da Coruña (UDC) acerca de la formación en TIC y más concretamente de la plataforma Moodle.

De los resultados obtenidos, podemos extraer una serie de conclusiones que nos permiten situarnos con mayor conocimiento ante las competencias digitales de alumnos/as y profesores para el uso de TIC y de la plataforma Moodle, y las necesidades de formación para el uso de estos recursos:

- La mayoría de los participantes consultados poseen en general conocimientos digitales para el uso de recursos tecnológicos e informáticos, aunque detectamos algunas diferencias entre profesorado y alumnado, ya que el primer 
colectivo posee mayor conocimiento para el manejo de recursos como cámaras de vídeo básicas, reproductores de vídeo e igualmente para el uso de distintas redes sociales, mientras que el segundo para el manejo de recursos y dispositivos de uso habitual como los de mensajería instantánea y chat, uso de cámaras de vídeo básicas y de distintas redes sociales. De modo contrario, tanto el profesorado como el alumnado manifestaron el poco conocimiento que poseen sobre los distintos componentes de un ordenador y diferentes dispositivos móviles. Consideramos tal y como indican Prendes y Gutiérrez (2013), que se debe ofrecer conocimiento sobre los conceptos y componentes básicos asociados a las TIC que propicien la puesta en práctica de acciones con estas tecnologías, que permitan resolver incidencias técnicas de forma autónoma y aprender nuevas herramientas, atendiendo las necesidades de docentes y alumnos.

- Detectamos diferencias significativas con relación a las competencias digitales que poseen profesorado y alumnado para el uso de TIC y de plataformas de teleformación. Mientras que los alumnos/as consideran tener mayores competencias a la hora de elaborar blogs, utilizar internet como medio didáctico y editar vídeos, el profesorado por su parte, se considera más competente a la hora de utilizar proyectores de presentaciones, realizar búsquedas en bases de datos y manejar plataformas virtuales de enseñanza-aprendizaje. Por el contrario, el alumnado indica a través de sus respuestas que es menos competente para el diseño de actividades para plataformas virtuales y de aplicaciones multimedia; siendo el diseño de páginas web sencillas, la elaboración de blogs y la edición de vídeos sencillos las competencias menos desarrolladas por parte del profesorado. Por lo tanto, los resultados de este estudio indican que existe la necesidad de capacitación de estas competencias tanto para el alumnado como para el profesorado.

- Existe discrepancia según lo expuesto por profesores y alumnos/as con relación a la valoración de la formación recibida para el uso de TIC por parte de la UDC, ya que la mayoría del profesorado participante considera que los conocimientos que poseen para el manejo de estas herramientas los han adquirido a través de cursos facilitados por la Institución, mientras que el alumnado manifestó totalmente lo contrario. Por lo tanto, sugerimos propuestas de formación en TIC para el alumnado, ya que como evidencia Sigalés (2004) la mejora continua de las competencias del alumno/a en el uso de estas herramientas permitirá una mayor flexibilización de las mismas y una mejora de la calidad de la docencia en procesos formativos.

- Detectamos un contraste en cuanto a la valoración que hace un colectivo sobre el contrario con relación a la formación para el uso de las TIC, ya que mientras que la mayoría del profesorado participante considera que el alumnado tiene un nivel de formación adecuado para el uso de estas herramientas, por el contrario, el alumnado considera que el profesorado tiene un menor nivel de formación. En España la implementación de los títulos de grado de Educación Infantil y Educación primaria, en el marco del EEES, supone una oportunidad ideal para poner en práctica una formación del profesorado en 
TIC que responda mejor a los desafíos de la educación del siglo XXI y que ofrezca más garantías de éxito en la integración curricular de las nuevas tecnologías en la educación obligatoria (Gutierrez, Palacios y Torrego, 2010).

- Los resultados de este estudio evidencian la necesidad de formación tanto de profesorado como del alumnado en competencias relacionadas con la "búsqueda de información" (revistas, documentos) y "el manejo y uso del ordenador". Consideramos que se deben fomentar espacios formativos en el ámbito de la UDC referido al uso de herramientas tecnológicas por parte de los profesores y alumnos, ya que como señalan Gutierrez, Palacios y Torrego (2010), para aumentar las posibilidades de éxito en el uso de las TIC como recurso didáctico se debe ofrecer una formación inicial y permanente del profesorado, tanto del futuro maestro como de los profesores universitarios encargados de su formación.

- Con relación a la plataforma Moodle los resultados de este estudio indican que tanto el profesorado como el alumnado consideran que no poseen los conocimientos necesarios para el uso adecuado de la misma, coincidiendo ambos colectivos al indicar que tanto docentes como alumnos/as deberían recibir formación para mejorar sus competencias en el manejo de la plataforma institucional. Por lo tanto, los resultados de este estudio indican que existe la necesidad de capacitación de competencias para el uso de la plataforma Moodle tanto en docentes como alumnos/as relacionadas con la subida y bajada de apuntes, el envío de documentos, el uso de chat/foro y la elaboración de exámenes.

Las evidencias derivadas de este estudio descriptivo han servido para determinar las necesidades formativas del profesorado y alumnado en TIC y en Moodle, constituyéndose las mismas como uno de los aspectos claves para lograr la integración exitosa de estos recursos en la práctica educativa diaria. De la misma forma, los resultados de este estudio han determinado igualmente como la UDC necesita continuar realizando esfuerzos con relación a la formación de su profesorado y alumnado, para lograr unos adecuados niveles de calidad en el proceso de E-A en el marco del EEES.

Finalmente, consideramos que la formación de los docentes y alumnos/as para la utilización de las TIC y de las plataformas de teleformación es hoy en día una necesidad incuestionable "si queremos incorporarlas de manera educativa y significativa a los procesos de enseñanza aprendizaje, y no meramente como un añadido que funcione independiente del resto de variables curriculares (contenidos, estrategias, metodologías,...)" (Cabero, Marín y Castaño, 2015, p. 13).

\section{REFERENCIAS BIBLIOGRÁFICAS}

Adell, J. (1997). Tendencias en educación en la sociedad de las tecnologías de la información. Revista electrónica de Tecnología Educativa, 4. Recuperado de http://www.aprender.org.ar/aprender/articulos/tecn-sociedad-informacion.htm.

Almerich, G., Suárez-Rodríguez, J. M., Belloch, C. y Bo, R. M. (2011). Las necesidades formativas del profesorado en TIC: perfiles formativos y elementos de 
complejidad. Revista Electrónica de Investigación y Evaluación Educativa, 17(2), 1-28. Recuperado de http://www.uv.es/RELIEVE/v17n2/RELIEVEv17n2_1.htm.

Álvarez, S., Cuéllar, C., López, B., Adrada, C., Anguiano, R., Bueno, A., Comas, I. y Gómez, S. (2011). Actitudes de los profesores ante la información de las TIC en la práctica docente. Estudio de un grupo de la Universidad de Valladolid. Edutec, 35. Recuperado de http://edutec.rediris.es/revelec2/revelec35/.

Area, M. (2005). Internet en la docencia universitaria. Webs docentes y aulas virtuales. Recuperado de http://webpages.ull.es/users/manarea/guiadidacticawebs.pdf.

Balanskat, A., Blamire, R. y Kefala, S. (2006). The ICT impact report. A review of studies of ICT impact on schools in Europe. European Schoolnet. Recuperado de http://insight.eun.org/shared/data/pdf/impact_study.pdf.

Banas, J. (2010). Teachers' Attitudes toward Technology. Considerations. Community \& Junior College Libraries, 16(2), 114-127.

Barberá, E. y Badia, A. (2008). Perspectivas actuales sobre la calidad educativa de los procesos de E-A que incorporan las TIC. En T. Barberá (Ed.). Cómo valorar la calidad de la enseñanza basada en las TIC (pp. 29-45). Barcelona: Graó.

Bates, T. (2005). Technology, E-learning and Distance Education. Abingdon: Routledge. BECTA (2004). A review of the Research Literature on Barriers to the Uptake of ICT by Teachers. Recuperado de http://partners.becta.org.uk/page_documents/research/ barriers.pdf.

Bisquerra, R. (Coord.) (2004). Métodos de investigación educativa. Madrid: La Muralla. Buendía, L., Colás, P. y Hernández, F. (1997). Métodos de investigación en psicopedagogía. Madrid: McGraw-Hill.

Cabero, J. (2004). Formación del profesorado en TIC. El gran caballo de batalla. Comunicación y Pedagogía: Nuevas tecnologías y recursos didácticos, 195, 27-31.

Cabero, J. (2014). Formación del profesorado universitario en TIC. Aplicación del método Delphi para la selección de los contenidos formativos. Educación XX1, 17(1), 1-132. DOI: http://doi.org/10.5944/educxx1.17.1.10707.

Cabero, J. y Marín, V. (2014). Miradas sobre la formación del profesorado en TIC. Enl@ ce Revista venezolana de Información, Tecnología y Conocimiento, 11(2), 11-24.

Cabero, J., Marín, V. y Castaño, C. M. (2015). Validación de la aplicación del modelo TPACK para la formación del profesorado en TIC. Recuperado de http://roderic. uv.es/bitstream/handle/10550/44762/5115993.pdf? sequence=1 \&isAllowed=y).

Condie, R. y Munro, B. (2007). The impact of ICT in schools: a landscape review. Becta research. Recuperado de http://www.becta.org.uk.

Diez, E. (2012). Modelos socioconstructivistas y colaborativos en el uso de las TIC en la formación inicial del profesorado. Revista de Educación, 358, 175-196. Recuperado de http://www.mecd.gob.es/dctm/revista-de-educacion/articulosre358/re35809.pdf?documentld=0901e72b8128205e.

Del Moral, E. y Villalustre, L. (2010). La formación del profesor 2.0. Desarrollo de competencias tecnológicas para la Escuela 2.0. Revista Miscelánea de Investigación, 23, 59-70.

Galanouli, D., Murphy, C. y Gardner, J. (2004). Teachers' perceptions of the effectiveness of ICT-competence training. Computers and Education, 43, 63-79. 
García, C., Días, P., Sorte, A., Díaz-Pérez, J., Leal, A. R. y Gandra, M. (2014). El uso de las TIC y herramientas de la Web 2.0 por maestros de la Educación Primaria y Educación Especial: La importancia de las competencias digitales. Profesorado. Revista de Currículum y formación del profesorado, 18(1), 241-255. Recuperado de http://www.ugr.es/ recfpro/rev181COL3.pdf.

Gilmore, E. (1998). Impact of Training on the Information Technology Attitudes of University Faculty. University of North Texas, Denton. Recuperado de http://www. tcet.unt.edu/research/dissert/gilmore/index.htm.

Gómez Galán, J. (2014). El fenómeno Mooc y la universalidad de la cultura: las nuevas fronteras de la Educación Superior. Profesorado. Revista de Currículum y formación del profesorado, 18(1), 241-255. Recuperado de http://www.ugr. es/ recfpro/rev181ART5.pdf.

Guarro, A. (2005). Los procesos de cambio educativo en una sociedad compleja. Diseño, desarrollo e innovación del currículum. Madrid: Pirámide.

Gutierrez, A., Palacios, A. y Torrego, L. (2010). La formación de los futuros maestros y la integración de las TIC en la educación: anatomía de un desencuentro. Revista de Educación, 352, 267-293. Recuperado de http://www.revistaeducacion. educacion.es/re352/re352_TIC.pdf.

Guzmán, T., García, M.T. y Chaparro, N. (2011). Formación docente para la integración de las TIC en la práctica educativa. Apertura, 3(1). Recuperado de http://www. udgvirtual.udg.mx/apertura/index. php/apertura3/article/view/181/196.

Henríquez, G. y Ugel, E. E. (2012). Migración de lo presencial a lo virtual en la asignatura introducción a la computación del programa de enfermería de la UCLA. Revista Iberoamericana de Educación a Distancia (RIED), 15(1), 127-142.

Hernández, G., García, C., y Navarrete, M. (2015). La inclusión de las TICS en el trabajo académico de los profesores universitarios. Revista Iberoamericana de Producción Académica y Gestión Educativa, 2, 1-13. Recuperado de http://cenid. org.mx/ctes_2015/memorias/index.php/ctes/article/view/184.

Jimoyiannis, A. y Komis, V. (2007). Examining teachers' beliefs about ICT in education. Implications of a teacher preparation programme. Teacher Development, 11(2), 149-173.

Llorente Cejudo, M. C. (2008). Aspectos fundamentales de la formación del profesorado en TIC. Pixel-Bit: Revista de Medios y Educación, 31, 121-130.

MacDowell, L. (2002). Electronic Information Resources in Undergraduate Education: an exploratory study of opportunities for student learning and Independence. British Journal of Educational Technology, 3(33), 255-266.

McMillan, J. y Schumager, S. (2005). Investigación educativa. Madrid: Pearson Addison Wesley.

Marín, F. y Amentia, J. I. (2009). Los estudiantes frente al reto de las TIC en la universidad. Moodle y eKasi en la Facultad de Ciencias Sociales y de la Comunicación (Universidad del País Vasco). Revista de estudios de comunicación, 27(14), 319-347.

Muñoz-Repiso, A. G. V., Gómez-Pablos, V. B., González, M. C., Martín, S. C., Rodero, L. M. G., Martín, A. H. y Marcos, J. J. M. (2015). La formación del profesorado 
universitario en Tecnologías de la Información y la Comunicación en la Universidad de Salamanca. RELATEC: Revista Latinoamericana de Tecnología Educativa, 14(1), 75-88.

Noelle, M. y Granados, J. (2015). Orientaciones para la formación didáctica del profesorado novel en las TIC: los entornos 2.0 y el trabajo colaborativo en el aula. Referencia Pedagógica, 1, 13-26. Recuperado de http://rrp.cujae.edu.cu/index. php/rrp/article/view/77.

Olcott, D. y Wright, S. (1995). An Institutional Support Framework for Increasing Faculty Participation in Postsecondary Distance Education. The American Journal of Distance Education, 9(3), 5-17.

Prendes, M., y Gutiérrez, I. (2013). Competencias tecnológicas del profesorado en las universidades españolas. Revista de Educación, 361, 196-222. Recuperado de http://www.mecd.gob.es/dctm/revista-de-educacion/articulosre361/re36108.pdf? documentld=0901e72b8162f031.

Rodríguez-Correa, M. y González-Sanmamed, M. (2013). La gestión del cambio institucional en las Universidades a través de las TIC. Revista de Docencia Universitaria, 11(3), 363-384.

Rodríguez-Correa, M., Iseni, A. y Varela, J. (2013). Virtual learning in higher education. Anglisticum Journal, 2(4), 262-278.

Salinas, J. (2002). Las TIC como medios para una nueva universidad. Efectos de la introducción de las TIC en la mejora de la docencia universitaria. Universitat de les Illes Balears. Recuperado de http://gte.uib.es/publicacions/CIDUI_salinas.pdf.

Sánchez, J., Sánchez, P. y Ramos, F. J. (2012). Usos pedagógicos de Moodle en la docencia universitaria desde la perspectiva de los estudiantes. Revista Iberoamericana de Educación, 60, 15-38.

Sang, G., Valcke. M., van Braak, J., Tondeur, J. y Zhu, Ch. (2011). Predicting ICT integration into classroom teaching in Chinese primary schools: exploring the complex interplay of teacher-related variables. Journal of Computer Assisted Learning, 27, 160-17.

Sigalés, C. (2004). Formación universitaria y TIC: nuevos usos y nuevos roles. Revista de Universidad y Sociedad del Conocimiento, 1(1), 1-6.

Scheaffer, R. L., Mendenhall, W. y Lyman, O. (2007). Elementos de muestreo. Madrid: Paraninfo.

Selwyn, N., Marriott, N. y Marriott, P. (2002). Home Computers \& University ICT Use. Journal of Computer Assisted Learning, 1(18), 44-45.

Usher, E. L. y Pajares, F. (2008). Self-efficacy for self-regulated learning: A validation study. Educational and Psychological Measurement, 68(3), 443-463.

Waite, S. (2004). Tools for the job: a report of two surveys of information and communications technology training and use for literacy in primary schools in the West of England. Journal of Computer Assisted Learning, 20, 11-20. 